\title{
A New Simulation-Optimization Approach using Hybrid Radial Basis Function and Particle Swarm Optimization in Multi-Transmitter Placement Planning
}

\author{
Amir Parnianifard 1, Muhammad Saadi ${ }^{2}$, Manus Pengnoo ${ }^{1}$, Muhammad Ali Imran ${ }^{3}$, and \\ Lunchakorn Wuttisittikulkij ${ }^{1, *}$ \\ 1 Department of Electrical Engineering, Faculty of Engineering, Chulalongkorn University, Bangkok 10330, \\ Thailand; amir.p@chula.ac.th \\ 2 Department of Electrical Engineering, University of Central Punjab, Lahore, Pakistan \\ 3 School of Engineering, University of Glasgow, Glasgow, G12 8QQ, UK \\ * Correspondence: lunchakorn.w@chula.ac.th;
}

\begin{abstract}
With the every passing day, the demand for data traffic is increasing and this demand forces the research community not only to look for alternating spectrum for communication but also urges the radio frequency planners to use the existing spectrum smartly. Cell size is shrinking with the every upcoming communication generation which makes the base station placement planning complex and cumbersome. In order to make the next-generation cost-effective, it is important to design the network in such a way which utilizes minimum number of base stations while ensure coverage and quality of service. This paper aims at develop a new approach using hybrid metaheuristic and metamodel applied in multi-transmitter placement planning (MTPP) problem. We apply radial basis function (RBF) metamodel to assist particle swarm optimizer (PSO) in a constrained simulation-optimization (SO) of MTPP to mitigate the associated computational burden of optimization procedure. We evaluate the effectiveness and applicability of proposed algorithm in a case study by simulating MTPP model with two, three, four and five transmitters.
\end{abstract}

Keywords: Simulation-Optimization; Radial Basis Function; Particle Swarm Optimization; MultiTransmitter Placement.

\section{Introduction}

The demands for data traffic are increasing at an exponential rate during the last decade and this trend is expected to continue in the future as well. Billions of humans and devices require highquality wireless communication in indoor and outdoor environments and this demand results in the evolution of heterogeneous networks (HetNets) with small cell size [1]. The solution is the deployment of more stationary or mobile base stations (BS) to meet the ever-increasing traffic demand [2]. With the rapid advancement in solid-state lighting (SSL) and microelectromechanical system, the research community can develop sensors which have the capability of sensing, computation and/or decision making thus expediting the utilization of wireless sensor networks (WSNs) and Internet of things (IoT) with many potential applications such as surveillance, automation, energy-saving, wildfire monitoring, smart cities, connected health to name a few [3].

Legacy networks have huge cell size, however, in next-generation networks, the cell size is dramatically reducing which creates a prime challenge for telecommunication network operators to scrupulously plan their complex network. 5G and beyond networks necessitate the massive deployment of BS. If these deployments are unplanned, this can result in a greater cost, higher interference levels, and overall straitened network performance. It is therefore important to do BS planning in such a manner that requires the least number of transmitters to accomplish the desired 
coverage ratio while maximizing the average received power in conjunction with the quality of service (QoS) [4]. Therefore, BS placement in a mobile network is crucial to effectively use the resources in next-generation networks, thus reducing the infrastructure cost. One of the challenges which is associated with the optimal placement of BS is the computational complexity of transmitter location optimization in contrast with the manual transmitter placement through site surveying [5].

One of the important communication design problems is the placement of base stations which gives optimal signal coverage and maintains the quality of service. In literature, various techniques have been proposed in order to accomplish this task. One such work that uses iterative heuristic search (IHS) to find the least number of transmitters and then a hierarchical simplex search algorithm to optimize their location to maximize the coverage, has been proposed which results in near-optimal transmitter placement [6]. The exact solutions are cost and computationally inefficient. To overcome the issues faced by evolutionary algorithms (EA) that require huge search space dimensions, a correlation approach was proposed in [7] that uses mutual interference parameters to form a group and this approach achieves better results than random-grouping based EA algorithms. The existing algorithms for optimal BS placement can be divided into heuristic, meta-heuristic and exact approaches. As heuristic approaches are problem dependent, however, meta-heuristic approaches are problem independent and GA is a typical example of a meta-heuristic approach. For millimeter wave frequencies supporting $5 \mathrm{G}$ services, swarm intelligence was used to find the minimum number of $\mathrm{BS}$ required while meeting the data rate requirements and then iterative method was used to remove the redundant BS. The resulting approach turns out to be an efficient one as the outage probability is quite low [8]. A novel geometry-induced genetic algorithm was proposed in [9] and gives promising results. The main approach used in that work was to divide the whole region of interest into sub-regions and then the local coverage ratio was ensured to be higher to give better performance.

In this study, we propose a new approach combining metaheuristic and metamodels applied in the practice of simulation-optimization for wireless signal coverage in the MTPP problem. Among metaheuristic techniques, PSO has attracted wide attention in engineering design problems due to its algorithmic simplicity and powerful search performance [10], [11]. However, the PSO algorithm that requires a large number of fitness evaluations before locating the global optimum, are often prevented from being applied to computationally expensive real-world problems [12]. Here, we assist PSO metaheuristic using RBF metamodel in an optimization procedure to alleviate the associated computational burden. RBF has become a widely accepted metamodel for deterministic and stochastic simulation metamodeling regarding its promising results particularly in solving complex simulation-based optimization problems [13]-[16].

The rest of this paper is organized as follows. The Section 2 provides methods and algorithmic framework of the proposed algorithm which has been developed for the MTPP problem. In Section 3, a numerical case study is served to show the applicability and effectiveness of the proposed algorithm in the simulation-optimization of the MTPP problem. Finally, this paper is concluded in Section 4.

\section{Materials and Methods}

\subsection{Metamodel based simulation-optimization}

Nowadays, developing processes in an engineering world is strongly associated with computer simulations. These computer codes can collect appropriate information about the characteristics of engineering problems before actually running the process. Computer simulations can help a rapid investigation of various alternative designs to decrease the required time to improve the system. besides, most numerical analyses for engineering problems make a well-suited use of mathematical programming. Clearly, a SO becomes necessary to find more interest and popularity than other optimization methods, to the complexity of many real-world optimization problems in way of mathematical formulation analyzing [17]-[20]. The main goals of simulation can be defined as two, first what-if study of model or sensitivity analysis, and second is optimization and validation of 
model [21]. The essential benefit of simulation is its ability to cover complex processes, either deterministic or random while eliminating mathematical sophistication [22]. In general, SO techniques are classified into model-based and metamodel-based approaches (also called surrogate methods) [23], [24].

In model-based techniques, the simulation running is not expensive and model output can be used directly in optimization. The common classical model-based approaches include metaheuristics (e.g. genetic algorithm (GA) [25], ant colony optimization (ACO) [26], and PSO [27]). These methods are more popular not only among computer scientists but also scientists from different fields due to some reasons such as simplicity, flexibility, derivation-free mechanism, and local optima avoidance [28]. It should be noted that in the model-based optimization methods, the original function is not expensive in terms of running (function evaluation) and model output can be used directly in optimization. Moreover, for "expensive optimization" such as computer-aided design optimization or simulation-based optimization using computer experiments with high computational cost (computational time consuming), classical optimization methods may perform poorly or may even fail to obtain a satisfactory solution within the allocated computer time. Moreover, to address such a challenge, metamodels have been applied in the literature [29]-[32].

Metamodeling techniques have been used to avoid intensive computational and numerical simulation models, which might squander time and resource for estimating the model's parameters. Metamodeling has utilized a variety of statistical and mathematical approaches for interpreting parameters and their relationship in an original model. The general overview of a metamodel is illustrated in Figure 1 [20]. Some main advantages of applying metamodel in optimization include i) improving the efficiency of optimization, ii) supporting parallel computation, iii) cover sensitivity analysis of input variables and gain better insights from the problem, and iv) handle both types of variables included discrete and continuous [30]. There are different metamodels have been developed in engineering practice such as an artificial neural network (ANN), RBF, support vector regression (SVM), Kriging (also known as Gaussian process), polynomial regression and multivariate adaptive regression splines [30], [31], [33].

\subsection{Radial Basis Function}

The RBF is a kind of neural network that employs RBF as a transfer function, see Figure 2, and is consisting of an input layer, hidden radial basis layer, and an output linear layer [34]. The RBF method for multivariate approximation is one of the common approaches in modern approximation methodologies. The RBF employs a linear combination of independent symmetric functions based on the Euclidean distances to compute the approximation function of response.

The simple mathematical forms of RBF can be expressed as:

$$
\widehat{f(x)}=\sum_{i=1}^{m} w_{i} h_{i}(x)
$$

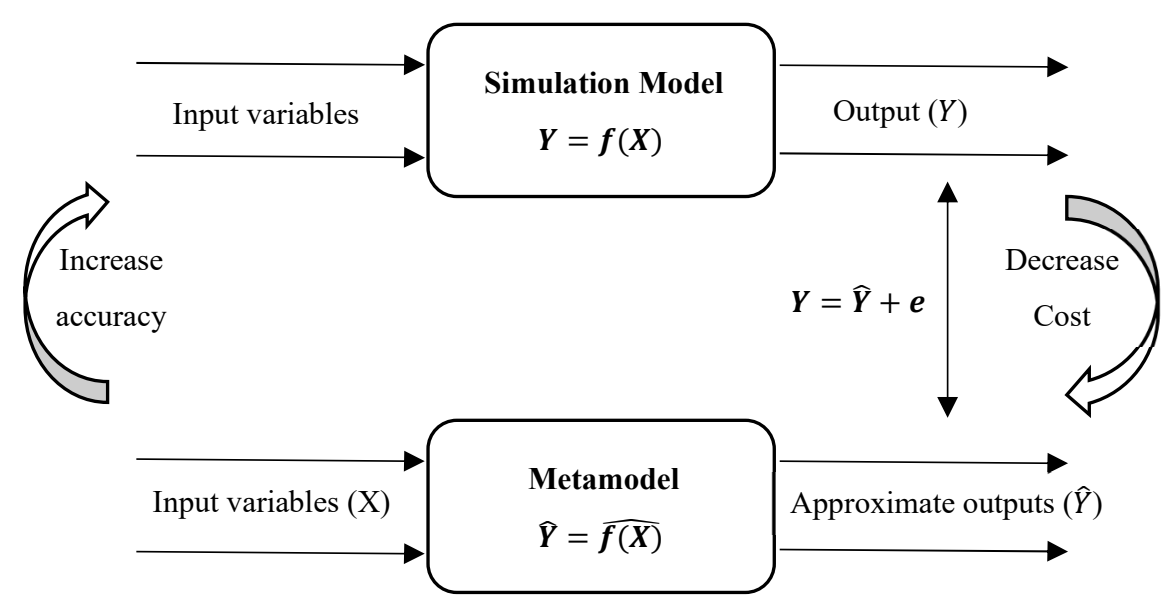

Figure 1. The viewpoint of metamodel and relationship with a simulation model. 


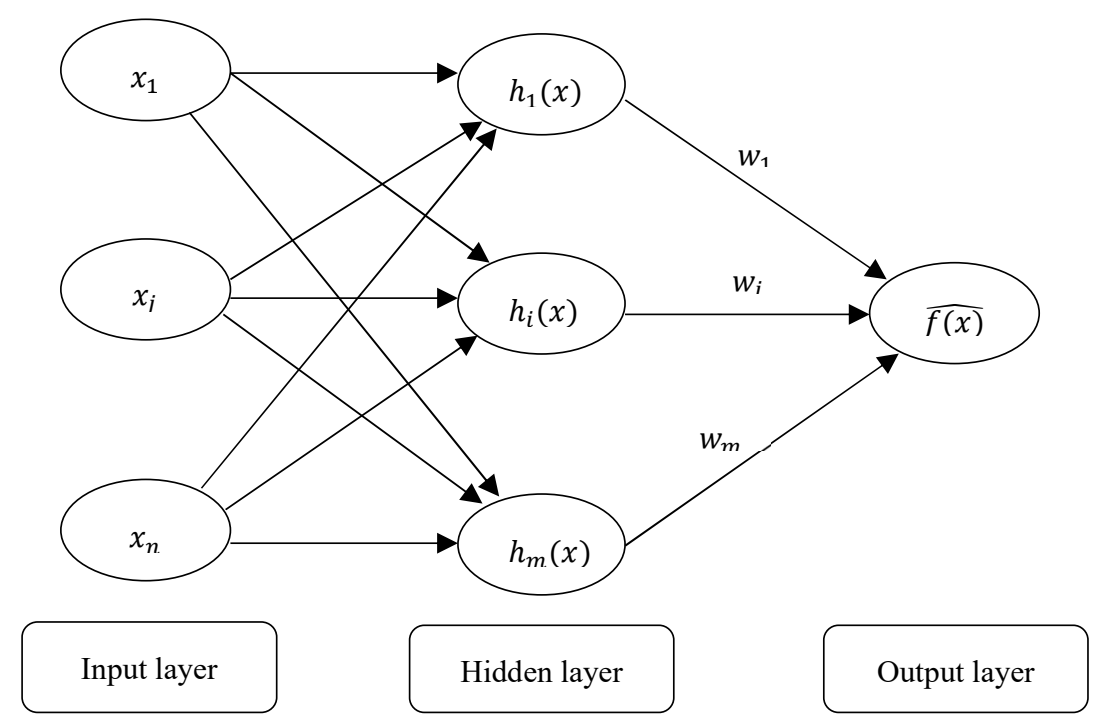

Figure 2. Radial basis function neural network.

where $w_{i},(i=1+2+\cdots+m)$ is the weight coefficients that need to be estimated, $m$ is number sample points, and $h_{i}(x)$ is $i$ th radial function. There are different types of RBF such as linear, cubic, Gaussian, multi-quadratic, and so on [34]. For instance, the common Gaussian form of the radial function is:

$$
h_{i}(x)=\exp \left(-r_{i}^{2}\right)
$$

where $r_{i}=\left\|x-x_{c i}\right\|$ and $x_{c i}$ is the center of $i$ th observed input. The value of weights parameters $w_{i}$ can be estimated by minimizing the sum csquare error of model:

$$
\text { Minimize } S S E=\sum_{i=1}^{m}\left(f\left(x_{i}\right)-\hat{y}_{i}\right)^{2}
$$

where $f\left(x_{i}\right)$ is exact output from the original model for $i$ th sample points, and $\hat{y}_{i}$ is related predicted output from RBF. Due to, the RBF interpolate the sample points, the SSE is expected to be $S S E \approx 0$.

\subsection{Particle Swarm Optimization}

The canonical PSO algorithm that simulates swarm behaviors of social animals such as bird flocking or fish schooling was proposed by [27]. The parameters of PSO consist of the number of particles, position of agent in the solution space, velocity and neighborhood of agents (communication of topology). The PSO algorithm begins by initializing the population first. The second step is calculating the fitness values of each particle, followed by updating individual and global bests, and later, the velocity and the position of the particles get updated. The second to fourth steps get repeated until the termination condition is satisfied [35], [36]. The PSO algorithm is formulated as follows [27], [35], [36]:

$$
\begin{gathered}
v_{i d}^{t+1}=v_{i d}^{t}+c_{1} \cdot \operatorname{rand}(0,1) \cdot\left(p_{i d}^{t}-x_{i d}^{t}\right)+c_{2} \cdot \operatorname{rand}(0,1) \cdot\left(p_{g d}^{t}-x_{i d}^{t}\right) \\
\text { and } \quad x_{i d}^{t+1}=x_{i d}^{t}+v_{i d}^{t}
\end{gathered}
$$

where $v_{i d}^{t}$ and $x_{i d}^{t}$ are particle velocity and particle position respectively. $d$ is the dimension in the search space, $i$ is the particle index, and $t$ is the iteration number. Expressions $c_{1}$ and $c_{2}$ represent the speed, regulating the length when flying towards the most optimal particles of the whole swarm and the most optimal individual particle. The term $p_{i}$ is the best position achieved so far by particle $i$ and $p_{g}$ is the best position found by the neighbors of particle $i$. The expression $\operatorname{rand}(0,1)$ shows the random values between 0 and 1 . The exploration happens if either or both of the differences between the particle's best $\left(p_{i d}^{t}\right)$ and previous particle's position $\left(x_{i d}^{t}\right)$, and between the population's all-time best $\left(p_{g d}^{t}\right)$ and previous particle's position $\left(x_{i d}^{t}\right)$ are large, and exploitation occurs when these values are both small. PSO has attracted wide attention in control engineering design problems due to its algorithmic simplicity and powerful search performance [10], 
[11]. However, PSO algorithm that requires a large number of fitness evaluations before locating the global optimum, are often prevented from being applied to computationally expensive real-world problems [12]. Therefore, metamodel-assisted PSO metaheuristic optimization algorithms have been interested in the literature, see [12], [37], [38].

\subsection{Algorithmic framework}

The algorithmic framework of proposed promising SO technique based on hybrid RBF metamodel and PSO metaheuristic is sketched in the following steps:

Input: Simulation Input/Output (I/O) set of data. Here, for the transmitters localization problem the input data set include $(X, Y)$ locations and output data set include average signal coverage (ASC) regarding each location.

Output: Estimation of Pareto frontier including a set of optimal locations for multiple transmitters.

\section{Step 1: Fit RBF metamodel over set of I/O data set.}

Step 2: Construct the objective function of optimization model.

Note: In the current paper in the MTPP problem, we consider the maximization of ASC as an objective function of the model. However, to smooth the amount of signal overlap by transmitters, we control the Euclidean distances between every two transmitters in a set of constraints. Let $k=1,2, \ldots, K$ is a number of transmitters in a model, therefore the proposed optimization model based on mathematical programming framework is constructed as below:

$$
\text { Maximize } A S C=\sum_{k=1}^{K} C\left(X_{k}, Y_{k}\right)
$$

Subject to:

$$
\begin{aligned}
& d\left(T_{i}, T_{j}\right) \geq \beta \frac{\|h\|}{K} \quad \text { for }\{\forall i, j \in(1,2, \ldots, K) \mid i<j\} \\
& 0 \leq X_{k} \leq u_{X} \quad, \quad 0 \leq Y_{k} \leq u_{Y}
\end{aligned}
$$

where $C_{k}(X, Y)$ is RBF estimation of ASC in location $(X, Y)$ for $k$ th transmitter. The terms $u_{X}$ and $u_{Y}$ indicate the length and width of the design space. The expression $d\left(T_{i}, T_{j}\right)=d\left(T_{j}, T_{i}\right)=\left\{\left(X_{i}-X_{j}\right)^{2}+\right.$ $\left.\left(Y_{i}-Y_{j}\right)^{2}\right\}^{\frac{1}{2}}$ shows the Euclidean distance (norm) between two locations of $i$ th and $j$ th transmitters. The expression $\|h\|$ shows the maximum Euclidean distance between two locations in the design space. The weight scale $\beta$ is defined by the decision maker and indicates the emphasis of the model over the distances between transmitters to smooth overall signal coverage. From Eq.(5) the optimal result depends on the value of $\beta$ which can be chosen by the decision-maker. Varying $\beta$ provides the capture of Pareto frontier (also called Pareto optimal efficiency). In fact, the set of optimal solutions obtained from fluctuating $\beta$ in the model, provides an estimate of the Pareto frontier [39]. As the aforementioned mathematical model is constrained (see Eq.(6)), a constraint handling method should be integrated into the optimization algorithm. There are several methods of constraints handling in the literature such as penalty functions, special operators, repair algorithms, separation of objectives and constraints, and hybrid methods [40]. In this study, we use the simplest method called the death penalty. In this method, search agents that violate any of the constraints with any level are treated as the same and penalized by assigning a large fitness value (small objective value in case of maximization). This method is very cheap and readily applicable to the PSO algorithm without algorithm modifications [40], [41].

Step 3: Run PSO optimizer for the constructed optimization model in the previous step and obtain the optimal result.

Step 4: Repeat three previous steps for different values of $\beta$ in Eq.(6) to obtain the estimation of Pareto frontier.

\section{Simulation-Based Optimization of MTTP: Case Study}

In this section, the applicability of the proposed algorithm in the MTPP problem is studied to obtain the optimal locations of each transmitter to maximize the amount of signal coverage. In 
continue, first the application of the proposed algorithm using hybrid RBF metamodel and PSO metaheuristic integrated by mathematical programming in current MTPP optimization problem is explained, and then, the obtained results are discussed in details.

\subsection{MTPP simulation-optimization model}

We create a simulation model using Python, which simulates the environment of an office room to implement MTPP problem. The obstacles in the room are modeled according to the layout of the office equipment by creating a polygon of each object and placing it in various positions in the room. The calculations of coverage area are based on the assumption that the signal propagation is omnidirectional, the transmitter can transmit the signal around in two dimensions. The signal power is transmitted from the transmitter according to the path loss equation and is limited to the lowest signal power that the receiver can receive with the sensitivity threshold of the receiver. The value will be the farthest range the signal can be received, which is like the signal radius of each transmitter. Due to the ultra-high-frequency characteristics of the signal, the connection between the transmitter and receiver needs line of sight. Therefore, when there are obstacles between the signal paths, the signal is immediately blocked. Our simulation uses ray-tracing method calculations from the transmitter to the receiver by detecting the intersection of obstacles that are created. The coverage area of each transmitter, therefore, comes from the coverage radius minus the area where the signal is blocked by obstacles. To evaluate the coverage rate in a two-dimensional area, we divide the entire monitoring region into $222 \times 222$ pixels. We apply the binary model in this study, means set 0 for the area that the transmitter does not cover, and 1 for the area that transmitter successfully covers. We first analyze the set of images that gained from the simulation model in order to obtain the numerical set of I/O data include $X$ and $Y$ locations and relevant ASC. We use the Matlab ${ }^{\circledR}$ environment to analyze the data set. However, we use Matlab® "Deep learning" toolbox for constructing RBF metamodel, and "particle swarm optimization" toolbox to perform PSO global optimizer. We define both $X$ axis and $Y$ axis in the same range of $[0,222]$. Figure 3 shows the scatter plot for 12,544 different $(X, Y)$ locations and relevant average coverages for one transmitter. Notably, in the current simulation model, we assume zero coverage for areas with obstacles in the design space. Figure 4 illustrates the contour plot of signal coverage in two-dimensions of design space including obstacle areas with zero coverage (areas with dark blue color). From the initial simulation model by locating one transmitter, the maximum ASC is obtained in a location of $X=26$ and $Y=0$ with $A S C=0.4355$, see Figure 5 .

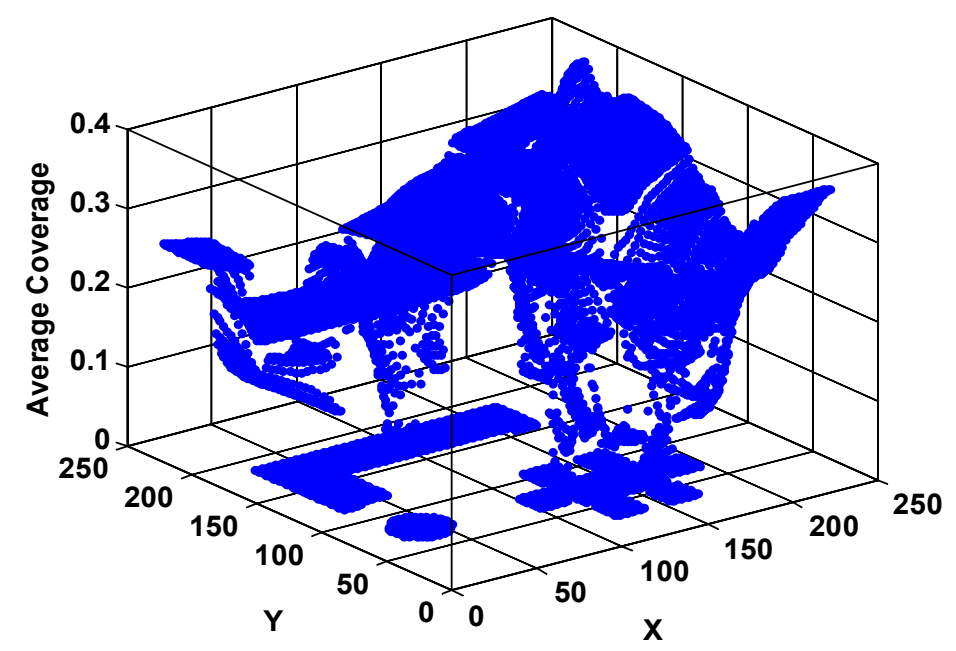

Figure 3. Three-dimensions scatter plot for different $(X, Y)$ transmitter locations and relevant average signal coverage. 


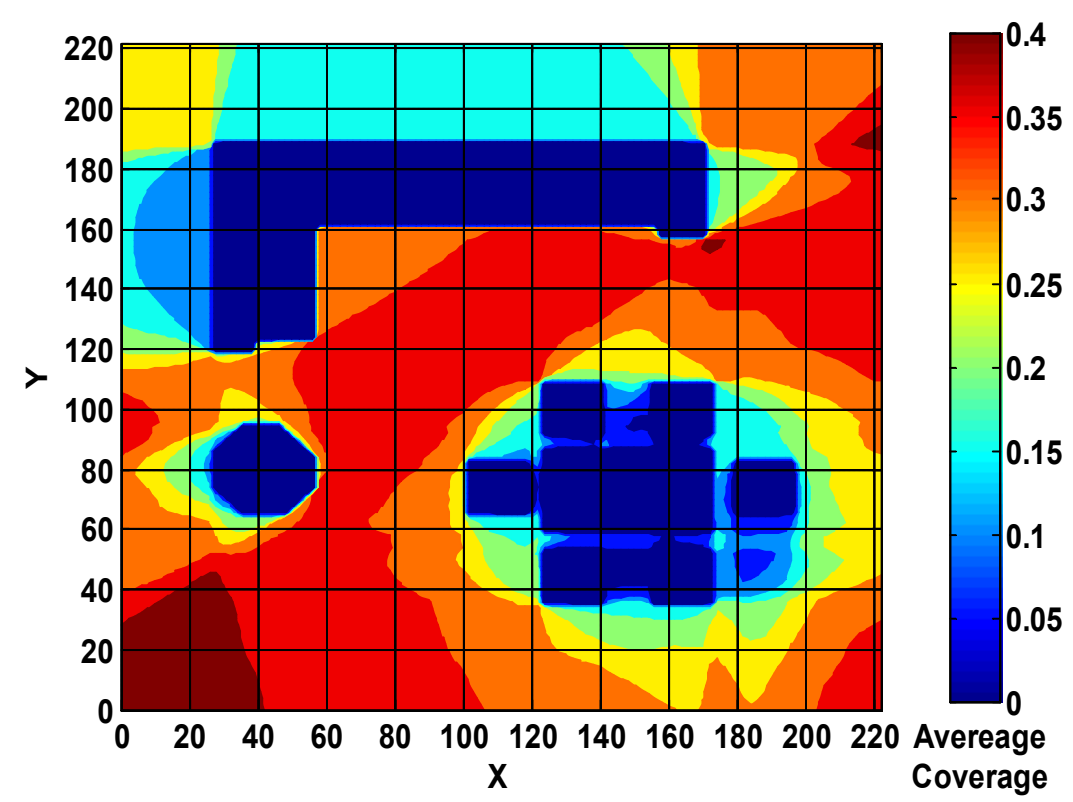

Figure 4. Contour plot for average coverage using one transmitter in all locations of design space. Obstacles areas show in dark blue color with zero signal coverage.

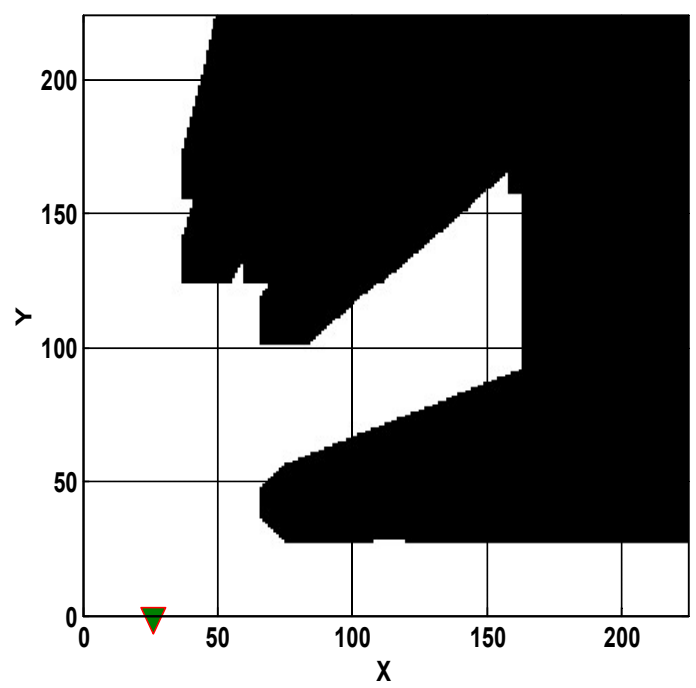

Figure 5. Maximum signal coverage in the model with one transmitter is obtained in $X=26, Y=0$ with $A S C=0.4355$. Black areas include obstacles and not covered by transmitter.

This paper aims to develop a new method to investigate the optimal locations of multiple transmitters. In the current instance, we apply the proposed algorithm for the MTPP problem with two, three, four and five transmitters separately. For this purpose, we first construct RBF metamodel over set I/O data obtained from the simulation model. We use the "newrbe" Matlab® function to construct RBF metamodel. Figure 6 shows the surface plot of constructed RBF metamodel and scatter plot of relevant mean square error of prediction.

Next, we construct the mathematical programming model for the MTPP problem (see Eq.(5) till Eq.(7)) with two, three, four, and five transmitters $(K=2,3,4,5)$ in a model separately. The design ranges for both $X$ axis and $Y$ axis are $0 \leq X_{k} \leq 222$ and $0 \leq Y_{k} \leq 222$ respectively. The $\|h\|$ is the maximum distance between two locations that here is the square diameter in design space and is equal to $\|h\|=\left\{(222-0)^{2}+(222-0)^{2}\right\}^{\frac{1}{2}}=313.956$. We repeat the optimization model in each 


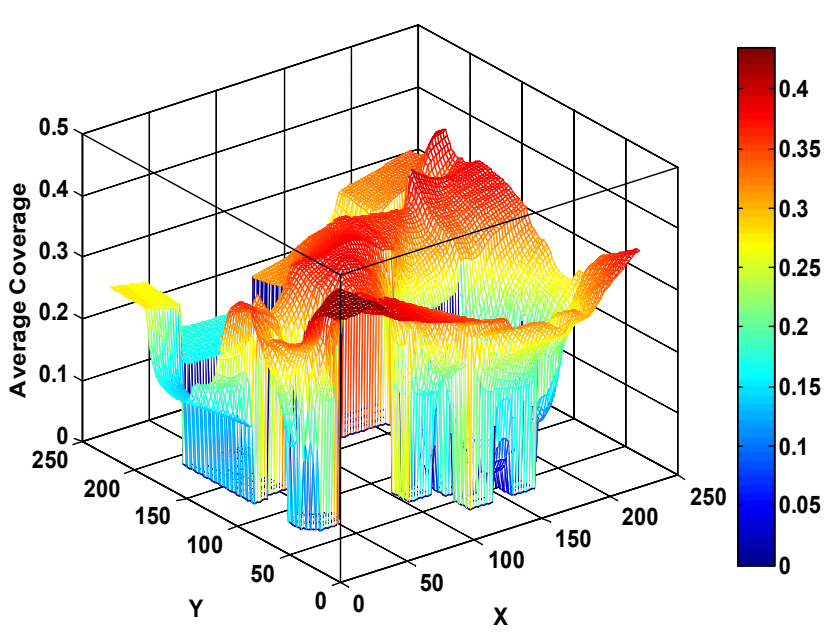

(a)

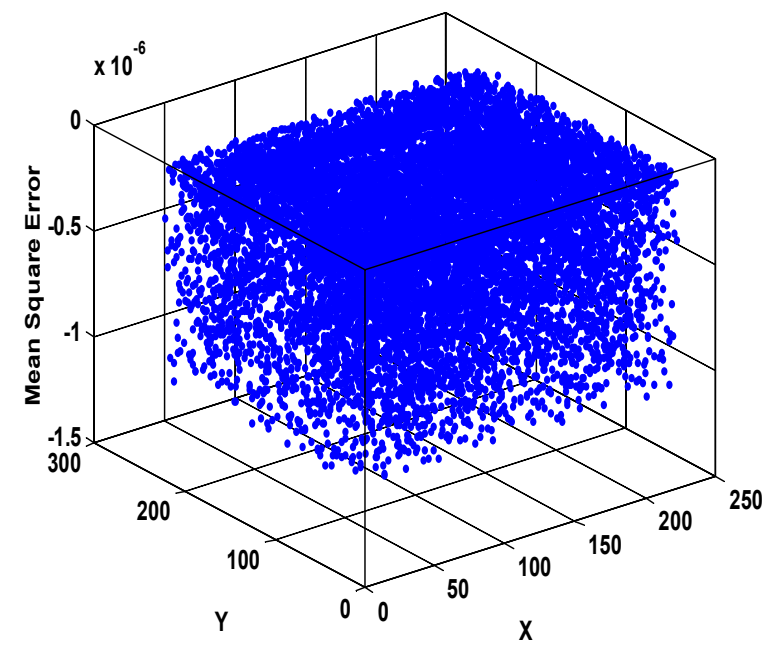

(b)

Figure 1 Surface plot of fitted RBF metamodel (a) and scatter plot for mean square error of constructed RBF prediction (b).

$K=2,3,4,5$ three times for $\beta=0.5, \beta=1$, and $\beta=1.5$, see Eq.(6). However, we run PSO optimizer for each optimization model separately. As, we use the RBF metamodel instead of the original simulation model, so we don't worry about the number of function evaluations (iterations) by PSO global optimizer. In the following, the obtained results for each combination of the MTPP optimization model are discussed.

\subsection{Results and discussion}

In the model with two transmitters $(K=2)$, we apply the proposed algorithm by integrating RBF metamodel which constructed over a set of I/O data (i.e. I/O data obtained from simulation model), and PSO optimizer. The optimization results in the model with two transmitters for three different values of $\beta$ (see Eq.(6)) are shown in Table 1. Figure 7 provides the schematic signal coverage in the design space when two transmitters located on their computed optimal locations. As can be seen, the highest $A S C$ is obtained by $\beta=0.5$ eqaul to 0.733 . The optimization procedure for both $\beta=1$ and $\beta=1.5$ provide the same results (same locations for two transmitters) with $A S C=$ 0.713 .

Next, we construct the optimization procedure based on the proposed hybrid RBF and PSO algorithm for such a case with three transmitters in the model. The obtained optimization results are shown in Table 2 for three different $\beta$ values and schematic coverages on optimal locations of transmitters are illustrated in Figure 8. As can be seen from Table 2, the highest signal coverage is obtained the same for $\beta=0.5$ and $\beta=1$ by $A S C=0.746$ when three transmitters are optimally located in the design space. With the same procedure, the proposed optimization algorithm is performed for the model with four transmitters and obtained results depicted in Table 3 and Figure 9. For such a case with four transmitters, the highest signal coverage in the model is obtained by $A S C=0.802$ in $\beta=1$ and followed closely by $\beta=0.5$ with $A S C=0.794$.

Table 1 The optimal locations and relevant overall signal coverage for two-transmitters localization model.

\begin{tabular}{|c|c|c|c|c|c|c|c|c|c|}
\hline \multirow{2}{*}{ Transmitter } & \multicolumn{3}{|c|}{$\beta=0.5$} & \multicolumn{3}{|c|}{$\beta=1$} & \multicolumn{3}{|c|}{$\beta=1.5$} \\
\hline & $\mathbf{X}$ & $\mathbf{Y}$ & ASC & $\mathbf{X}$ & $\mathbf{Y}$ & ASC & $\mathbf{X}$ & $\mathbf{Y}$ & ASC \\
\hline$\# 1$ & 26 & 0 & 0.435 & 0 & 0 & 0.426 & 0 & 0 & 0.426 \\
\hline$\# 2$ & 222 & 188 & 0.407 & 222 & 188 & 0.407 & 222 & 188 & 0.407 \\
\hline Overall Coverage & \multicolumn{3}{|c|}{0.733} & \multicolumn{3}{|c|}{0.713} & \multicolumn{3}{|c|}{0.713} \\
\hline
\end{tabular}



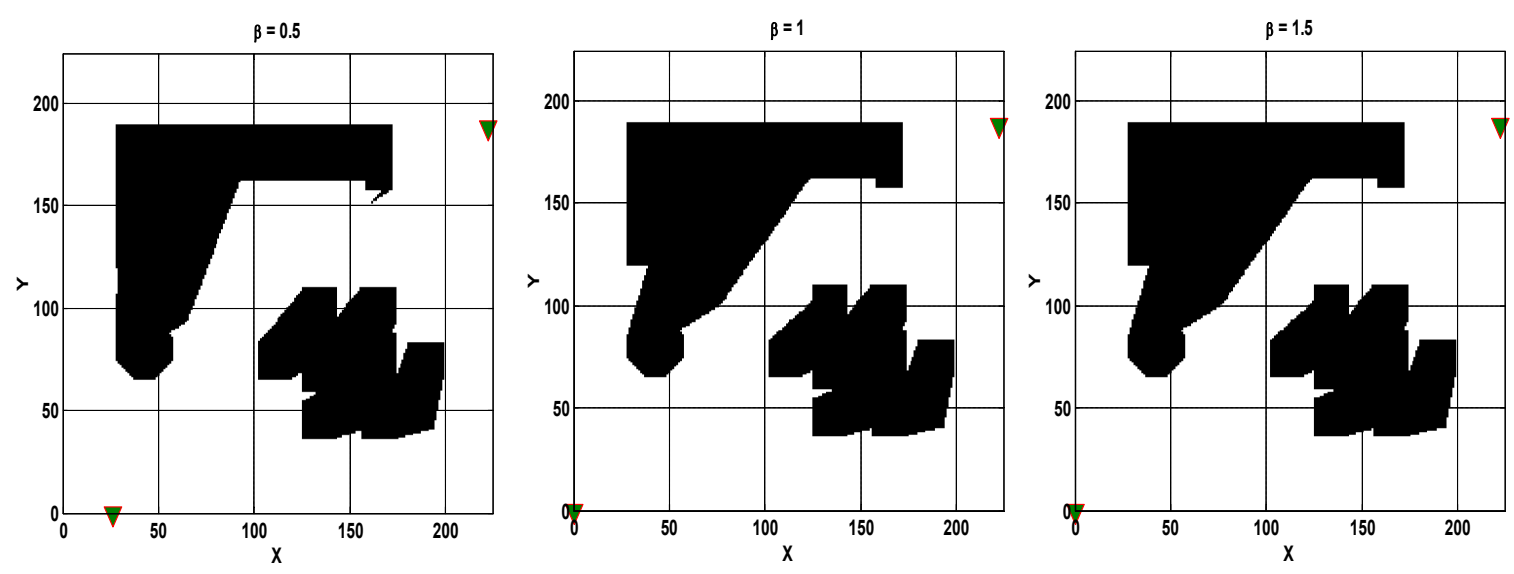

Figure 7. Optimal locations (i.e. are shown by triangle marker) and overall signal coverage of two transmitters in MTL problem for $\beta=0.5, \beta=1$, and $\beta=1.5$. Black areas include obstacles and not covered by transmitters.

Finally, the application of the proposed algorithm is used for the MTPP problem with five transmitters. Optimal locations and relevant obtained results are provided in Table 4 and schematically are shown in Figure 10. The obtained results for optimization of a model with five transmitters reveal that the highest signal coverage is obtained in $\beta=1$ by $A S C=0.802$ the same as model with four transmitters. At the end, we compare all obtained results based on $\beta=0.5, \beta=$ 1 and $\beta=1.5$ as shown in Figure 11. It is clear that the $\beta=0.5$ shows the good results for a model with two, three, and four transmitters expect for a model with five transmitters. However, due to considered obstacle areas in the original simulation model, the model with five transmitters is depicted very close behavior in covering design space with a model using four transmitters.

\section{Conclusions}

Simulation-optimization is found to be a viable solution for solving complex real-world problems like the base station placement problem in 5G and beyond networks. The existing algorithms for optimal BS placement can be broadly classified into three categories namely heuristic, meta-heuristic, and exact approaches. Out of these three approaches, the meta-heuristic approach is considered to be the most suited as its fast convergence to find optimal solution. In this paper, we combine the PSO metaheuristic with RBF metamodel to propose a computationally less complex algorithm for solving MTPP optimization problem with two, three, four and five transmitters. The obtained results show the effectiveness and applicability of proposed algorithm.

Supplementary Materials: Matlab ${ }^{\circledR}$ codes and functions constructed through the development of the proposed algorithm are available online at www.mdpi.com/xxx/s1.

Author Contributions: All authors have contributed to this study. All authors have read and agreed to the published version of the manuscript.

Funding: This research project is supported by Second Century Fund (C2F), Chulalongkorn University, Bangkok.

Table 2. The optimal locations and relevant overall signal coverage for three-transmitters localization model.

\begin{tabular}{|c|c|c|c|c|c|c|c|c|c|}
\hline \multirow{2}{*}{ Transmitter } & \multicolumn{3}{|c|}{$\beta=0.5$} & \multicolumn{3}{|c|}{$\beta=1$} & \multicolumn{3}{|c|}{$\beta=1.5$} \\
\hline & $\mathbf{X}$ & $\mathbf{Y}$ & ASC & $\mathbf{X}$ & $\mathbf{Y}$ & ASC & $\mathbf{X}$ & $\mathbf{Y}$ & ASC \\
\hline$\# 1$ & 26 & 0 & 0.435 & 222 & 0 & 0.384 & 0 & 0 & 0.426 \\
\hline$\# 2$ & 222 & 188 & 0.407 & 222 & 188 & 0.407 & 222 & 188 & 0.407 \\
\hline$\# \mathbf{3}$ & 222 & 0 & 0.384 & 26 & 0 & 0.435 & 222 & 0 & 0.384 \\
\hline Overall Coverage & \multicolumn{3}{|c|}{0.746} & \multicolumn{3}{|c|}{0.746} & \multicolumn{3}{|c|}{0.726} \\
\hline
\end{tabular}



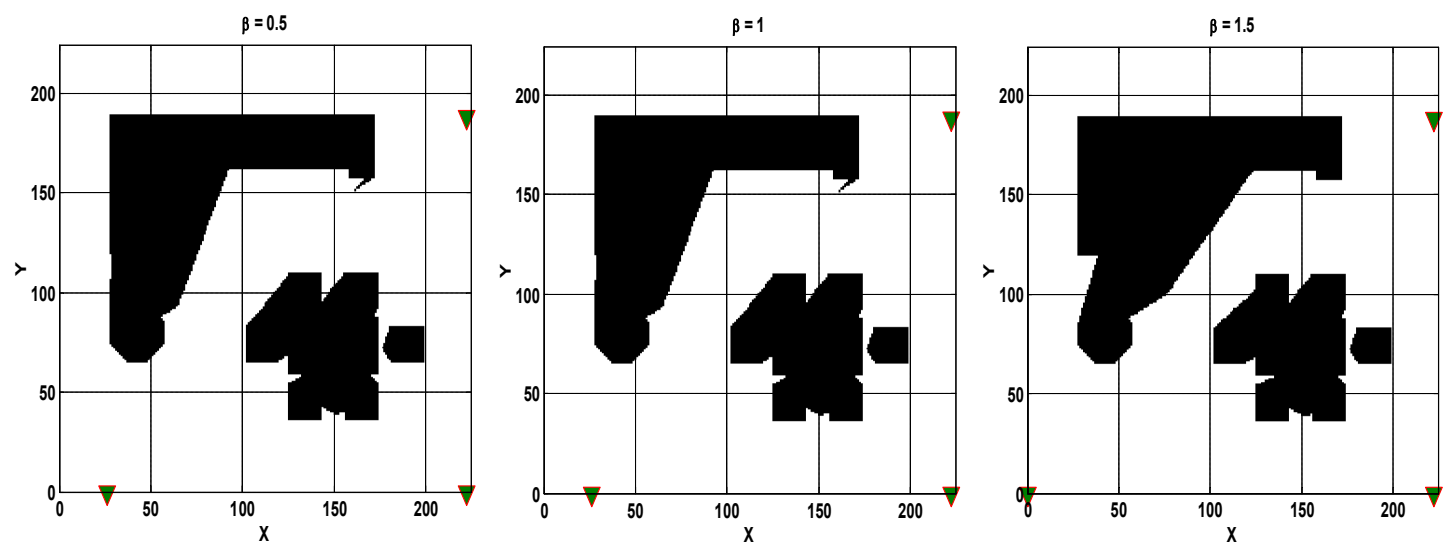

Figure 8. Optimal locations (i.e. are shown by triangle marker) and overall signal coverage of three transmitters in MTL problem for $\beta=0.5, \beta=1$, and $\beta=1.5$. Black areas include obstacles and not covered by transmitters.

Table 3. The optimal locations and relevant overall signal coverage for four-transmitters localization model.

\begin{tabular}{|c|c|c|c|c|c|c|c|c|c|}
\hline \multirow{2}{*}{ Transmitter } & \multicolumn{3}{|c|}{$\beta=0.5$} & \multicolumn{3}{|c|}{$\beta=1$} & \multicolumn{3}{|c|}{$\beta=1.5$} \\
\hline & $\mathbf{X}$ & $\mathbf{Y}$ & ASC & $\mathbf{X}$ & $\mathbf{Y}$ & ASC & $\mathbf{X}$ & $\mathbf{Y}$ & ASC \\
\hline$\# 1$ & 222 & 148 & 0.386 & 222 & 0 & 0.384 & 222 & 188 & 0.407 \\
\hline$\# 2$ & 222 & 188 & 0.407 & 74 & 92 & 0.389 & 26 & 0 & 0.435 \\
\hline$\# 3$ & 222 & 0 & 0.384 & 0 & 0 & 0.426 & 0 & 222 & 0.277 \\
\hline$\# 4$ & 0 & 0 & 0.426 & 222 & 188 & 0.407 & 222 & 0 & 0.384 \\
\hline
\end{tabular}

Overall Coverage

0.794

0.802

0.748
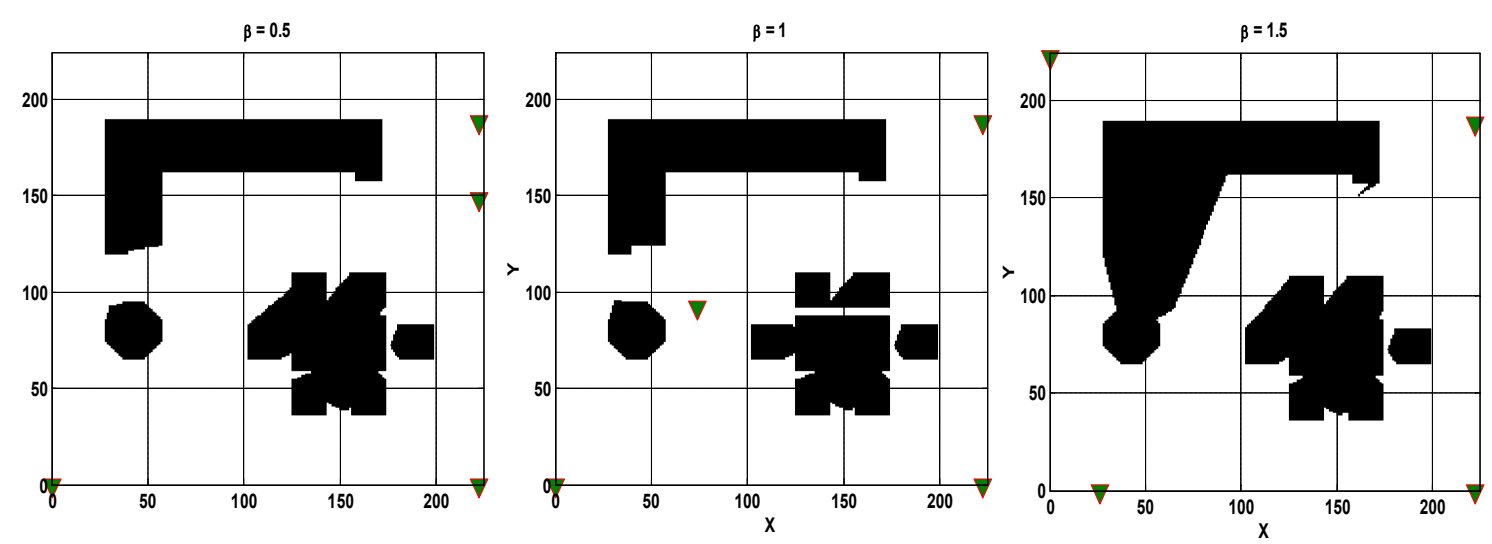

Figure 9. Optimal locations (i.e. are shown by triangle marker) and overall signal coverage of four transmitters in MTL problem for $\beta=0.5, \beta=1$, and $\beta=1.5$. Black areas include obstacles and not covered by transmitters.

Table 4. The optimal locations and relevant overall signal coverage for five-transmitters localization model.

\begin{tabular}{|c|c|c|c|c|c|c|c|c|c|}
\hline \multirow{2}{*}{ Transmitter } & \multicolumn{3}{|c|}{$\beta=0.5$} & \multicolumn{3}{|c|}{$\beta=1$} & \multicolumn{3}{|c|}{$\beta=1.5$} \\
\hline & $\mathbf{X}$ & $\mathbf{Y}$ & ASC & $\mathbf{X}$ & $\mathbf{Y}$ & ASC & $\mathbf{X}$ & $\mathbf{Y}$ & ASC \\
\hline$\# 1$ & 0 & 0 & 0.426 & 222 & 188 & 0.407 & 222 & 0 & 0.384 \\
\hline$\# 2$ & 222 & 188 & 0.407 & 0 & 0 & 0.426 & 222 & 188 & 0.407 \\
\hline$\# \mathbf{3}$ & 222 & 0 & 0.384 & 74 & 92 & 0.389 & 90 & 110 & 0.389 \\
\hline$\# 4$ & 26 & 32 & 0.427 & 222 & 0 & 0.384 & 0 & 0 & 0.426 \\
\hline$\# \mathbf{5}$ & 32 & 0 & 0.425 & 62 & 0 & 0.381 & 2 & 222 & 0.276 \\
\hline Overall Coverage & \multicolumn{3}{|c|}{0.759} & \multicolumn{3}{|c|}{0.802} & \multicolumn{3}{|c|}{0.799} \\
\hline
\end{tabular}



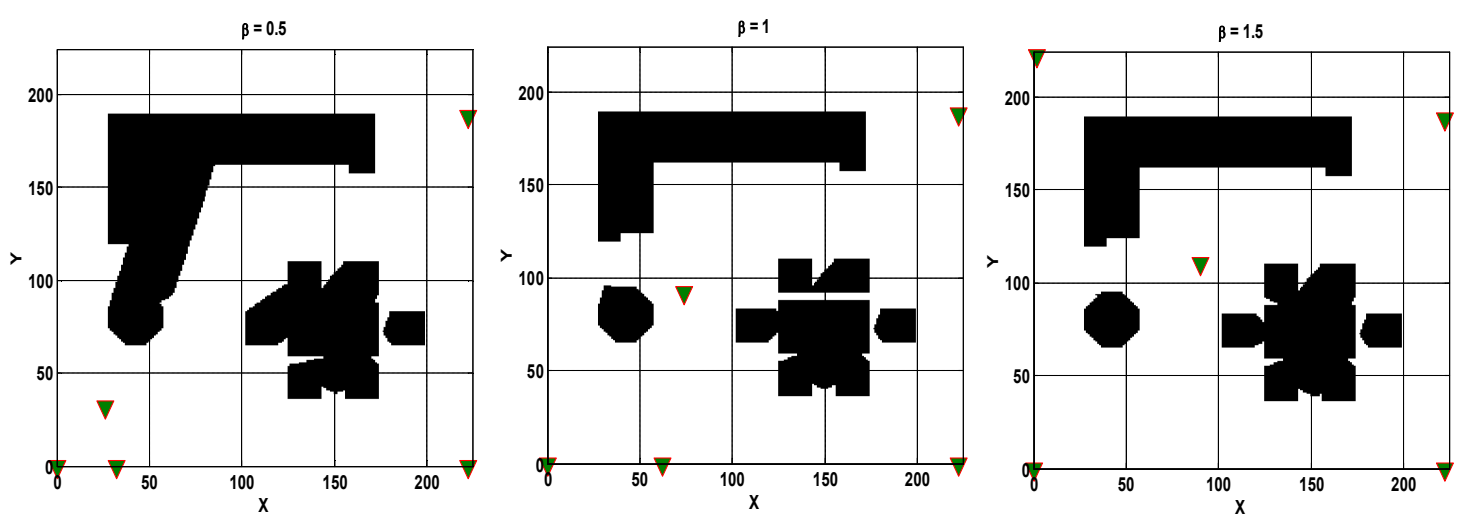

Figure 10. Optimal locations (i.e. are shown by triangle marker) and overall signal coverage of five transmitters in

MTL problem for $\beta=0.5, \beta=1$, and $\beta=1.5$. Black areas include obstacles and not covered by transmitters.

Acknowledgments: The authors acknowledge the support by Second Century Fund (C2F), Chulalongkorn University, Bangkok for funding and postdoctoral fellowship.

Conflicts of Interest: The authors declare no conflict of interest. The funders had no role in the design of the study; in the collection, analyses, or interpretation of data; in the writing of the manuscript, or in the decision to publish the results.

\section{References}

1. W. Ejaz, S. Kandeepan, and A. Anpalagan, Optimal placement and number of energy transmitters in wireless sensor networks for RF energy transfer, in 2015 IEEE 26th Annual International Symposium on Personal, Indoor, and Mobile Radio Communications (PIMRC), 2015, pp. 1238-1243.

2. F. Alduraibi, N. Lasla, and M. Younis, Coverage-based node placement optimization in wireless sensor network with linear topology, in 2016 IEEE International Conference on Communications (ICC), 2016, pp. $1-6$.

3. M. Saadi, M. T. Noor, A. Imran, W. T. Toor, S. Mumtaz, and L. Wuttisittikulkij, IoT Enabled Quality of Experience Measurement for Next Generation Networks in Smart Cities, Sustainable Cities and Society, p. 102266, 2020.

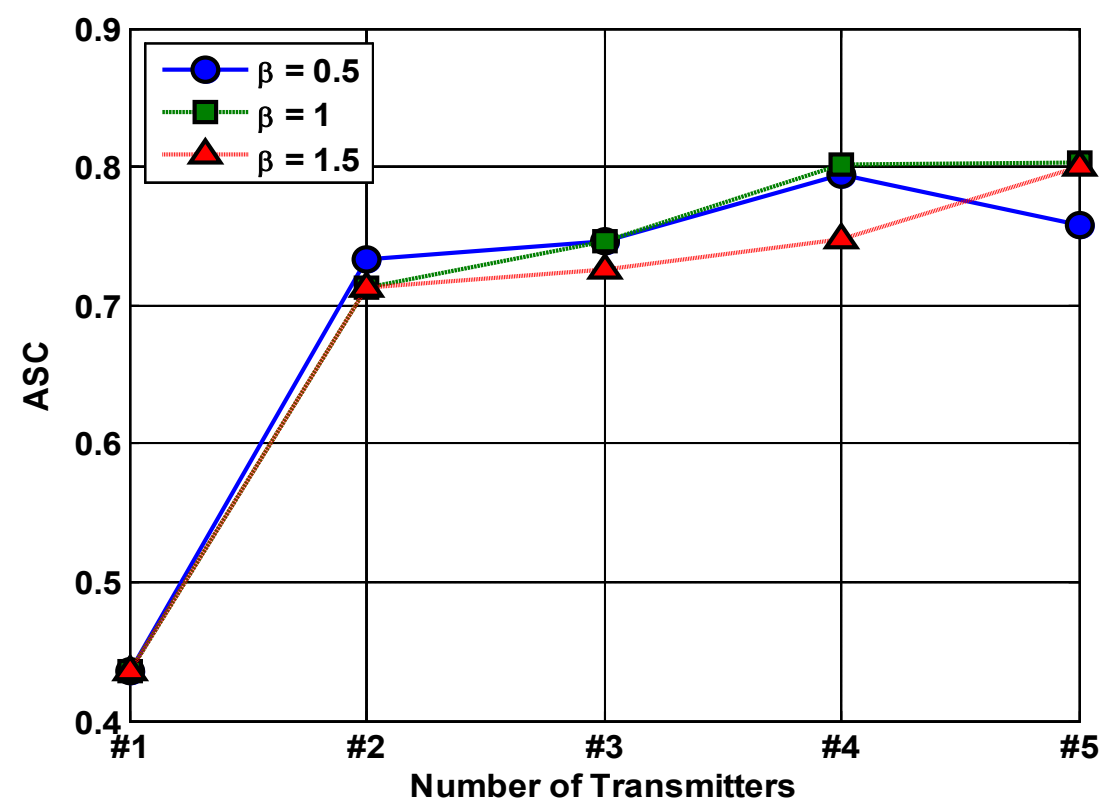

Figure 11. Average signal coverage for different number of transmitters in the model based on three different $\beta$ values. 
4. Y. Lin, W. Yu, and Y. Lostanlen, Optimization of wireless access point placement in realistic urban heterogeneous networks, in 2012 IEEE Global Communications Conference (GLOBECOM), 2012, pp. 49634968.

5. S. Yin, L. Li, and F. R. Yu, Resource allocation and basestation placement in downlink cellular networks assisted by multiple wireless powered UAVs, IEEE Transactions on Vehicular Technology, 69, (2), pp. 2171-2184, 2019.

6. H. Liang, B. Wang, W. Liu, and H. Xu, A novel transmitter placement scheme based on hierarchical simplex search for indoor wireless coverage optimization, IEEE transactions on antennas and propagation, 60, (8), pp. 3921-3932, 2012.

7. S. Lee, S. Lee, K. Kim, and Y. H. Kim, Base station placement algorithm for large-scale LTE heterogeneous networks, PloS one, 10, (10), p. e0139190, 2015.

8. H. Ganame, L. Yingzhuang, H. Ghazzai, and D. Kamissoko, 5G Base Station Deployment Perspectives in Millimeter Wave Frequencies Using Meta-Heuristic Algorithms, Electronics, 8, (11), p. 1318, 2019.

9. Y. Liu, W. Huangfu, H. Zhang, H. Wang, W. An, and K. Long, An Efficient Geometry-Induced Genetic Algorithm for Base Station Placement in Cellular Networks, IEEE Access, 7, pp. 108604-108616, 2019.

10. M. P. Aghababa, Optimal design of fractional-order PID controller for five bar linkage robot using a new particle swarm optimization algorithm, Soft Computing, 20, (10), pp. 4055-4067, 2016.

11. M. Zamani, M. Karimi-Ghartemani, N. Sadati, and M. Parniani, Design of a fractional order PID controller for an AVR using particle swarm optimization, Control Engineering Practice, 17, (12), pp. 1380-1387, 2009.

12. H. Yu, Y. Tan, J. Zeng, C. Sun, and Y. Jin, Surrogate-assisted hierarchical particle swarm optimization, Information Sciences, 454-455, pp. 59-72, 2018.

13. D. J. Fonseca, D. O. Navaresse, and G. P. Moynihan, Simulation metamodeling through artificial neural networks, Engineering Applications of Artificial Intelligence, 16, (3), pp. 177-183, 2003.

14. R. A. Kilmer, A. E. Smith, and L. J. Shuman, Computing confidence intervals for stochastic simulation using neural network metamodels, Computers \& Industrial Engineering, 36, (2), pp. 391-407, 1999.

15. M. Scardi, Advances in neural network modeling of phytoplankton primary production, Ecological Modelling, 2001. .

16. F. M. Alam, K. R. McNaught, and T. J. Ringrose, A comparison of experimental designs in the development of a neural network simulation metamodel, Simulation Modelling Practice and Theory, 12, (7-8 SPEC. ISS.), pp. 559-578, 2004.

17. S. Jacobson and L. Schruben, A review of techniques for simulation optimization, Cornell University Operations Research and Industrial Engineering, 1986.

18. J. R. Swisher, P. D. Hyden, S. H. Jacobson, and L. W. Schruben, A survey of simulation optimization techniques and procedures, in Simulation Conference, 2000. Proceedings. Winter, 2000, 1, pp. 119-128.

19. S. Amaran, N. V. Sahinidis, B. Sharda, and S. J. Bury, Simulation optimization: a review of algorithms and applications, Annals of Operations Research, 240, (1), pp. 351-380, 2016.

20. A. Parnianifard, A. Azfanizam, M. Ariffin, M. Ismail, and N. Ebrahim, Recent developments in metamodel based robust black-box simulation optimization: An overview, Decision Science Letters, 8, (1), pp. 17-44, 2019.

21. W. C. M. van Beers and J. P. C. Kleijnen, Kriging for interpolation in random simulation, Journal of the Operational Research Society, 54, (3), pp. 255-262, 2003.

22. G. Figueira and B. Almada-Lobo, Hybrid simulation optimization methods a taxonomy and discussion, Simulation Modelling Practice and Theory, 46, pp. 118-134, 2014.

23. F. A. C. Viana, T. W. Simpson, V. Balabanov, and V. Toropov, Metamodeling in Multidisciplinary Design Optimization: How Far Have We Really Come?, AIAA Journal, 52, (4), pp. 670-690, 2014.

24. A. Mohammad Nezhad and H. Mahlooji, An artificial neural network meta-model for constrained simulation optimization, Journal of the Operational Research Society, 65, (8), pp. 1232-1244, 2013.

25. E. Bonabeau, D. de R. D. F. Marco, M. Dorigo, and G. Theraulaz, Swarm intelligence: from natural to artificial systems, (1). Oxford university press, 1999.

26. M. Dorigo and M. Birattari, Ant colony optimization. Springer, 2010.

27. R. Eberhart and J. Kennedy, Particle swarm optimization, in Proceedings of the IEEE international conference on neural networks, 1995, 4, pp. 1942-1948.

28. S. Mirjalili, S. M. Mirjalili, and A. Lewis, Grey Wolf Optimizer, Advances in Engineering Software, 69, pp. 46-61, 2014. 
29. R. Jin, X. Du, and W. Chen, The use of metamodeling techniques for optimization under uncertainty, 25, (2). 2003.

30. G. Wang and S. Shan, Review of Metamodeling Techniques in Support of Engineering Design Optimization, Journal of Mechanical Design, 129, (4), pp. 370-380, 2007.

31. T. W. Simpson, J. D. Poplinski, P. N. Koch, and J. K. Allen, Metamodels for Computer-based Engineering Design: Survey and recommendations, Engineering With Computers, 17, (2), pp. 129-150, 2001.

32. A. Parnianifard, A. S. Azfanizam, M. K. A. Ariffin, and M. I. S. Ismail, An overview on robust design hybrid metamodeling: Advanced methodology in process optimization under uncertainty, International Journal of Industrial Engineering Computations, 9, (1), pp. 1-32, 2018.

33. A. Parnianifard, A. S. Azfanizam, M. K. A. Ariffin, and M. I. S. Ismail, Comparative study of metamodeling and sampling design for expensive and semi-expensive simulation models under uncertainty, SIMULATION, 96, (1), pp. 89-110, May 2019.

34. M. D. Buhmann, Radial basis functions: theory and implementations, 12. Cambridge university press, 2003.

35. M. N. Ab Wahab, S. Nefti-Meziani, and A. Atyabi, A Comprehensive Review of Swarm Optimization Algorithms, PLOS ONE, 10, (5), p. e0122827, May 2015.

36. Y. Del Valle, G. K. Venayagamoorthy, S. Mohagheghi, J.-C. Hernandez, and R. G. Harley, Particle swarm optimization: basic concepts, variants and applications in power systems, IEEE Transactions on evolutionary computation, 12, (2), pp. 171-195, 2008.

37. S. Dutta, A sequential metamodel-based method for structural optimization under uncertainty, Structures, 26, (July 2019), pp. 54-65, 2020.

38. R. G. Regis, Particle swarm with radial basis function surrogates for expensive black-box optimization, Journal of Computational Science, 5, (1), pp. 12-23, 2014.

39. K. Miettinen, Nonlinear multiobjective optimization, 12. Springer Science \& Business Media, 2012.

40. C. A. Coello Coello, Theoretical and numerical constraint-handling techniques used with evolutionary algorithms: a survey of the state of the art, Computer Methods in Applied Mechanics and Engineering, 191, (11), pp. 1245-1287, 2002.

41. S. Mirjalili, The ant lion optimizer, Advances in Engineering Software, 83, pp. 80-98, 2015. 